\title{
Effect of bending stiffness on the peeling behavior of an elastic thin film on a rigid substrate
}

\author{
Zhilong Peng and Shaohua Chen* \\ The State Key Laboratory of Nonlinear Mechanics, Institute of Mechanics, Chinese Academy of Sciences, Beijing 100190, China
}

(Received 22 September 2014; revised manuscript received 2 January 2015; published 3 April 2015)

\begin{abstract}
Inspired by the experimental observation that the maximum peeling force of elastic films on rigid substrates does not always emerge at the steady-state peeling stage, but sometimes at the initial one, a theoretical model is established in this paper, in which not only the effect of the film's bending stiffness on the peeling force is considered, but also the whole peeling process, from the initiation of debonding to the steady-state stage, is characterized. Typical peeling force-displacement curves and deformed profiles of the film reappear for the whole peeling process. For the case of a film with relatively large bending stiffness, the maximum peeling force is found arising at the initial peeling stage and the larger the stiffness of the film, the larger the maximum peeling force is. With the peeling distance increasing, the peeling force is reduced from the maximum to a constant at the steady-state stage. For the case of a film with relatively small stiffness, the peeling force increases monotonically at the initial stage and then achieves a constant as the maximum at the steady-state stage. Furthermore, the peeling forces in the steady-state stage are compared with those of the classical Kendall model. All the theoretical predictions agree well with the existing experimental and numerical observations, from which the maximum peeling force can be predicted precisely no matter what the stiffness of the film is. The results in this paper should be very helpful in the design and assessment of the film-substrate interface.
\end{abstract}

DOI: 10.1103/PhysRevE.91.042401

PACS number(s): $68.15 .+\mathrm{e}, 68.35 . \mathrm{Np}$

\section{INTRODUCTION}

Fundamental understanding of the peeling behaviors, debonding mechanisms, and influencing factors of the interface strength of film-substrate systems is very important for the wide applications of thin films in modern advanced materials and engineering and it also plays a significant role in material protecting, connecting, and strengthening [1,2]. The peel test, as a well-known technique to measure the adhesion properties and interface strength between a thin film and a substrate, was designed 60 years ago by Spies [2,3]. Due to its simplicity and easy operation, the peel test is one of the efficient methods for assessing the interface mechanical properties so far.

In the past few decades, peeling behaviors of thin films on substrates have been widely investigated experimentally, numerically, and theoretically $[1,4-8]$. Kendall's model, as a classically theoretical one, is very popular in analyzing the interface properties of elastic film-substrate systems [9], in which the peel-off force is found depending on the interface adhesion energy, the stretching strain energy stored in the film, and the peeling angle. As a pioneering work, Kendall's model was later widely extended to analyze interfacial adhesion mechanisms of elastic-plastic or viscoelastic thin films [5,7,10,11], heterogeneous thin films [12,13], films with pretension [14,15], large deformation of film [16], and bioinspired nanofilms $[15,17-19]$. Another representative theory for thin films peeling from substrates is the beam model, in which films are regarded as slender beams and the pure bending beam theory is adopted to analyze the interfacial bonding and debonding mechanism $[4,20,21]$. It was widely used to analyze interface properties of ductile thin films considering the plastic or viscoelastic energy dissipation $[5,22,23]$. Cohesive zone

*Corresponding author: chenshaohua72@ hotmail.com conception is also often introduced in applications of both representative models to analyze interfacial stress distributions as well as the adhesion energy in peel tests [24-26]. Systematic analyses of interface characteristics of film-substrate systems have been made by the above two types of models, but most of them focus on the steady-state peeling process, neglecting the peeling mechanisms before the film reaches the steady-state stage. It has been found experimentally that the peeling force always varies as a function of the peeling displacement and the maximum peeling force does not always appear at the steady-state peeling process but at the initial stage. The peeling force increases first at the initial peeling stage and then decreases to a constant in the steady-state peeling process [1,4,27]. In addition, finite element calculations carried out by Sauer [1] show that the film's end axis is not strictly consistent with the direction of the external peeling force before the steady-state peeling process is reached due to the bending stiffness of film. Oyharcabal and Frisch [28] used continuum mechanics to study theoretically the unbinding of an elastic inextensible rod from a smooth substrate but only considering the bending energy under a vertical applied force (a $90^{\circ}$ peeling angle). A systematic study of the description of the whole peeling process of a film with finite bending stiffness at an arbitrary peeling angle and prediction of precisely the maximum peeling force of the film-substrate system are lacking.

In order to answer the above question, a method for the minimum potential energy is adopted in the present paper with respect to the system of an elastic film adhering to a rigid substrate. The interaction between the elastic film and the rigid substrate is represented by the LennardJones (LJ) potential, which can describe different interaction energies by adjusting the corresponding parameters. The whole peeling process and the maximum peeling force will be analyzed as well as the exact profile of the deformed film. 
(a)
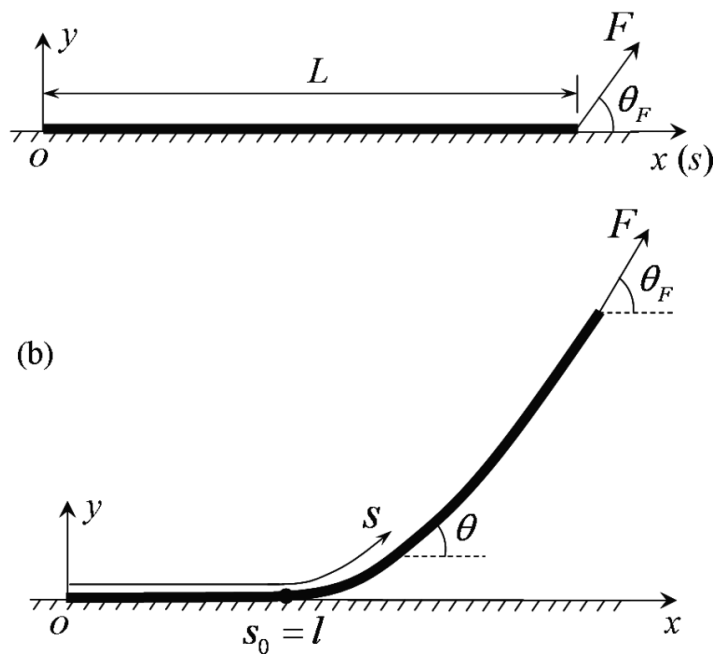

FIG. 1. Schematic of an elastic thin film with a length $L$ peeling from a rigid substrate with a peeling force $F$ and a peeling angle $\theta_{F}$ at the right end of the film. A curvilinear coordinate $(s, \theta)$ and a rectangular one $(x, y)$ are attached to the film-substrate system with the origin $o$ at the left end of the film. (a) The initial peeling state and (b) an intermediate state.

\section{THEORETICAL MODEL OF A THIN-FILM PEELING FROM A RIGID SUBSTRATE}

\section{A. Generalized peeling model}

A plane strain model of an elastic thin film adhering to a smooth rigid substrate is shown in Fig. 1(a), where a peeling force $F$ is acted on the right end of the film with a peeling angle $\theta_{F}$ and the interface adhesion is assumed to be a van der Waalslike interaction. With the increasing peeling force $F$, the elastic film will debond from the rigid substrate gradually as shown in Fig. 1(b); it reaches the steady-state peeling process when the film's axis direction at the right end is consistent with the peeling angle. Here $(s, \theta)$ and $(x, y)$ are curvilinear and rectangular coordinates with their origin $o$ at the left end of the film, respectively, with $d x=d s \cos \theta$ and $d y=d s \sin \theta$. Initially, the two coordinates overlap each other as shown in Fig. 1(a). In our analysis, we assume that the length of the film $L$ is long enough so that the steady-state stage can always be achieved before the film detaches from the substrate entirely. Young's modulus and the thickness of the thin film are denoted by $E$ and $h$, respectively.

The potential energy of the film-substrate system can be expressed as

$$
\begin{aligned}
E= & \int_{0}^{L} \frac{1}{2} D \theta^{\prime 2}(s) d s+\int_{0}^{L} \frac{1}{2} E \varepsilon^{2} h d s-\vec{F} \cdot \vec{u}_{F} \\
& -\int_{0}^{L} F \varepsilon d s+\int_{0}^{L} V(y) d s,
\end{aligned}
$$

where the first term on the right-hand side of Eq. (1) is the bending elastic energy, $D=h^{3} / 12$ is the bending stiffness of the film, $\theta$ is the tangential angle of each point on the film, and $s$ is the arc length of the film from the origin $o$. The second term on the right-hand side is the tension strain energy, with the elastic strain of the film defined as $\varepsilon=F \cos \left(\theta-\theta_{F}\right) / E h$.
The third term corresponds to the potential of the external force due to the displacement $\vec{u}_{F}$ of the loading point without considering the film's extension and the fourth terms is the work done by the external force due to tension of the elastic film. The displacement $\vec{u}_{F}$ can be expressed as

$$
\vec{u}_{F}=\int_{0}^{L}\left(\begin{array}{c}
\cos \theta \\
\sin \theta
\end{array}\right) d s .
$$

The last term on the right-hand side of Eq. (1) is the interaction potential between the film and the substrate, which depends on the separation between the elastic film and rigid substrate and is often described by the LJ potential $[28,29]$

$$
V(y)=W\left[\left(\frac{\sigma}{y}\right)^{9}-\left(\frac{\sigma}{y}\right)^{3}\right],
$$

where $W$ is a parameter determining the depth of the potential well, $\sigma$ denotes the effective interaction distance, which is often taken to be $0.2-0.3 \mathrm{~nm}$, and $y$ is the separation between the film and substrate with an equilibrium distance $y_{0}=\sqrt[6]{3} \sigma$. When $y \rightarrow \infty$, the interaction potential energy tends to be zero. The minimum interaction potential energy can be found at the equilibrium position, which takes a negative value.

The profile of the film can be described by $x^{\prime}=\cos \theta$ and $y^{\prime}=\sin \theta$, where $x^{\prime}$ and $y^{\prime}$ stand for derivatives with respect to the arc length $s$. Two Lagrange multipliers $\lambda_{1}$ and $\lambda_{2}$ have to be introduced due to the coupled dependence between $x$ (or $y$ ) and $\theta$. Then Eq. (1) can be rewritten as

$$
\begin{aligned}
E= & \int_{0}^{L} \frac{1}{2} D \theta^{\prime 2} d s+\int_{0}^{L} \frac{F^{2}}{2 E h} \cos ^{2}\left(\theta-\theta_{F}\right) d s \\
& -\int_{0}^{L} \frac{F^{2}}{E h} \cos \left(\theta-\theta_{F}\right) \cos \left(\theta_{L}-\theta_{F}\right) d s \\
& -\int_{0}^{L} F \cos \left(\theta-\theta_{F}\right) d s+\int_{0}^{L} W\left[\left(\frac{\sigma}{y}\right)^{9}-\left(\frac{\sigma}{y}\right)^{3}\right] d s \\
& +\int_{0}^{L} \lambda_{1}\left(y^{\prime}-\sin \theta\right) d s+\int_{0}^{L} \lambda_{2}\left(x^{\prime}-\cos \theta\right) d s .
\end{aligned}
$$

Due to the free bending moment at both ends of the film, we have the boundary conditions $\theta^{\prime}(0)=0$ and $\theta^{\prime}(L)=0$.

In order to find the equilibrium state of the film-substrate system and the profile of the film, the principle of the minimum potential energy is used in the present paper. Let the first variation of the potential energy in Eq. (4) with respect to $\theta$ equal zero,

$$
\begin{aligned}
\delta E= & -\int_{0}^{L}\left[D \theta^{\prime \prime}+\lambda_{1} \cos \theta-\lambda_{2} \sin \theta\right. \\
& \left.+\frac{F^{2}}{E h} \cos \left(\theta-\theta_{F}\right) \sin \left(\theta-\theta_{F}\right)-F \sin \left(\theta-\theta_{F}\right)\right] \delta \theta d s \\
& +\int_{0}^{L}\left[\frac{F^{2}}{E h} \sin \left(\theta-\theta_{F}\right) \cos \left(\theta_{L}-\theta_{F}\right)\right] \delta \theta d s \\
& -\int_{0}^{L}\left(\lambda_{1}^{\prime}+\frac{\partial V}{\partial y}\right) \delta y d s-\int_{0}^{L} \lambda^{\prime} \delta x d s \\
& +\int_{0}^{L}\left(y^{\prime}-\sin \theta\right) \delta \lambda_{1} d s+\int_{0}^{L}\left(x^{\prime}-\cos \theta\right) \delta \lambda_{2} d s \\
& +\left.D \theta^{\prime} \delta \theta\right|_{0} ^{L}+\left.\lambda_{1} \delta y\right|_{0} ^{L}+\left.\lambda_{2} \delta x\right|_{0} ^{L}=0 .
\end{aligned}
$$


Combining the above boundary conditions yields

$$
\begin{gathered}
D \theta^{\prime \prime}+\lambda_{1} \cos \theta-\lambda_{2} \sin \theta+\frac{F^{2}}{E h} \cos \left(\theta-\theta_{F}\right) \sin \left(\theta-\theta_{F}\right) \\
-F \sin \left(\theta-\theta_{F}\right)-\frac{F^{2}}{E h} \sin \left(\theta-\theta_{F}\right) \cos \left(\theta_{L}-\theta_{F}\right)=0, \\
\lambda_{1}^{\prime}=-\frac{\partial V}{\partial y}, \quad \lambda_{2}=0, \\
x^{\prime}=\cos \theta, \quad y^{\prime}=\sin \theta, \\
\lambda_{1}(L)=0, \quad \theta^{\prime}(0)=0, \quad \theta^{\prime}(L)=0 .
\end{gathered}
$$

Equations (6) and (7) are the equilibrium ones for the film. Equation (8) indicates the geometrical profile of the film and Eq. (9) reproduces the natural boundary conditions. One can see that Eqs. (6)-(9) belong to a typical boundaryvalue problem of nonlinear ordinary differential equations. An analytical solution is not easy to achieve and numerical calculations will be used with a standard shooting method. The detailed results will be analyzed in the following section.

\section{B. Approximate peeling model}

In the above general peeling model, the interface energy is governed by the LJ potential in the whole length of the film. As a result, the interacting energy exists between the film and the rigid substrate, though it may be very small for a relatively large separation between them. Similar to the classical Kendall model, we assume here that the interface interaction vanishes in the peeled-off region with a length $L-l$ and the left region
$[0, l]$ of the film adheres to the rigid substrate perfectly with a constant interface adhesion energy $\Delta \gamma$. Then, only the adhesion energy in the region $[0, l]$ contributes to the total energy of the system, while the peeled-off part $[l, L]$ should undergo bending and stretching as shown in Fig. 1(b). Such a simplified model is often used to analyze the adhesion between a film (or a cantilever beam) and a substrate [12,28,30,31].

The potential energy of the film-substrate system in the simplified model can be expressed as

$$
\begin{aligned}
E= & \int_{l}^{L} \frac{1}{2} D \theta^{\prime 2} d s+\int_{l}^{L} \frac{1}{2} E \varepsilon^{2} h d s-\vec{F} \cdot \vec{u}_{F} \\
& -\int_{l}^{L} F \varepsilon d s-\int_{0}^{l} \Delta \gamma d s \\
= & \int_{l}^{L} \frac{1}{2} D \theta^{\prime 2} d s+\int_{l}^{L} \frac{F^{2}}{2 E h} \cos ^{2}\left(\theta-\theta_{F}\right) d s \\
& -\int_{0}^{L} F \cos \left(\theta-\theta_{F}\right) d s \\
& -\int_{l}^{L} \frac{F^{2}}{E h} \cos \left(\theta-\theta_{F}\right) \cos \left(\theta_{L}-\theta_{F}\right) d s-\int_{0}^{l} \Delta \gamma d s,
\end{aligned}
$$

where the adhesion energy $\Delta \gamma$ can be obtained from Eq. (3), $\Delta \gamma=\left|V\left(y_{0}\right)\right|=2 \sqrt{3} W / 9$. Here a positive adhesion energy is defined.

For the present model, the bonding length $l$ is a variable that depends on the peeling force $F$. Let the first variation of the potential energy in Eq. (10) with respect to $\theta$ and $l$ equal zero,

$$
\delta E=\delta E_{1}+\delta E_{2}=0
$$

where

$$
\begin{aligned}
\delta E_{1}= & -\int_{l}^{L}\left[D \theta^{\prime \prime}+\frac{F^{2}}{E h} \cos \left(\theta-\theta_{F}\right) \sin \left(\theta-\theta_{F}\right)-F \sin \left(\theta-\theta_{F}\right)\right] \delta \theta d s \\
& +\int_{l}^{L}\left[\frac{F^{2}}{E h} \sin \left(\theta-\theta_{F}\right) \cos \left(\theta_{L}-\theta_{F}\right)\right] \delta \theta d s+\left.D \theta^{\prime} \delta \theta\right|_{l} ^{L}
\end{aligned}
$$

and

$$
\delta E_{2}=\left.\left[\frac{1}{2} D \theta^{\prime 2}-\frac{F^{2}}{2 E h} \cos ^{2}\left(\theta-\theta_{F}\right)+\frac{F^{2}}{E h} \cos \left(\theta-\theta_{F}\right) \cos \left(\theta_{L}-\theta_{F}\right)\right]\right|_{S=l} \delta l-\Delta \gamma \delta l .
$$

Considering the boundary conditions $\theta(l)=0$ and $\theta^{\prime}(L)=0$ leads to

$$
\begin{gathered}
D \theta^{\prime \prime}+\frac{F^{2}}{E h} \cos \left(\theta-\theta_{F}\right) \sin \left(\theta-\theta_{F}\right)-F \sin \left(\theta-\theta_{F}\right)-\frac{F^{2}}{E h} \sin \left(\theta-\theta_{F}\right) \cos \left(\theta_{L}-\theta_{F}\right)=0, \\
\frac{1}{2} D \theta_{l}^{\prime 2}-\frac{F^{2}}{2 E h} \cos ^{2} \theta_{F}+\frac{F^{2}}{E h} \cos \theta_{F} \cos \left(\theta_{L}-\theta_{F}\right)-\Delta \gamma=0 .
\end{gathered}
$$

Multiplying $\theta^{\prime}$ on both sides of Eq. (14) and then integrating it from $l$ to $L$, we can simplify Eq. (14) with the help of Eq. (15) as

$$
\frac{F^{2}}{2 E h} \cos ^{2}\left(\theta_{L}-\theta_{F}\right)+F\left[\cos \left(\theta_{L}-\theta_{F}\right)-\cos \theta_{F}\right]-\Delta \gamma=0 .
$$


From Eq. (16) one can obtain the peeling force with a closed-form solution

$$
F=\frac{-E h\left[\cos \left(\theta_{L}-\theta_{F}\right)-\cos \theta_{F}\right]+\sqrt{E^{2} h^{2}\left[\cos \left(\theta_{L}-\theta_{F}\right)-\cos \theta_{F}\right]^{2}+2 E h \Delta \gamma \cos ^{2}\left(\theta_{L}-\theta_{F}\right)}}{\cos ^{2}\left(\theta_{L}-\theta_{F}\right)} .
$$

When the peeling process reaches the steady-state stage, the axis direction of the film at the loading end will be consistent with the one of the peeling force, i.e., $\theta_{L}=\theta_{F}$. Therefore, Eq. (17) can be reduced to

$$
F=\frac{2 \Delta \gamma}{\sqrt{\left(1-\cos \theta_{F}\right)^{2}+2 \Delta \gamma / E h}+\left(1-\cos \theta_{F}\right)} .
$$

It is interesting to find that the present model is identical to the classical Kendall peeling model at the steady-state peeling process.

The adhering length $l$ of the film-substrate system in an equilibrium state can be obtained as a function of the peeling force from Eqs. (14) and (15) as

$$
l=L-\int_{0}^{\theta(L)} \frac{d \theta}{\sqrt{(2 / D)\left(\Delta \gamma-C_{1}+C_{2}\right)}},
$$

where

$$
C_{1}=F\left[\cos \left(\theta-\theta_{F}\right)-\cos \theta_{F}\right]
$$

$$
C_{2}=\frac{F^{2}}{2 E h} \cos ^{2}\left(\theta-\theta_{F}\right)-\frac{F^{2}}{E h} \cos \left(\theta-\theta_{F}\right) \cos \left(\theta_{L}-\theta_{F}\right) .
$$

The profile of the elastic film under the peeling force can be given implicitly as

$$
\begin{aligned}
S & =L-\int_{\theta(S)}^{\theta(L)} \frac{d \theta}{\sqrt{(2 / D)\left(\Delta \gamma-C_{1}+C_{2}\right)}}, \quad S \geqslant l, \\
\theta(S) & =0, \quad 0<S<l .
\end{aligned}
$$

\section{RESULTS AND DISCUSSION}

The simplified (approximate) model and the general one are compared with the peeling angle $\theta_{F}=90^{\circ}$, which exhibits an inconsistence at the initially peeling stage and the transient one as shown in Fig. 2. It is found that the peeling force of the simplified model decreases monotonically with the increase of $y_{L}$; in particular, the maximum force $F=\sqrt{2 E h \Delta \gamma} / \cos \theta_{F}$ emerges at $y_{L}=0$ (or $\theta_{L}=0$ ) with $l=L$, which is totally contradictory to the general model.

What leads to the inconsistence, especially the initial varying trend of the peeling force in two models? The main reason is the different shapes of the released domain of the film in the two models, leading to different peeling responses.

Different shapes of the released domain of the film are influenced by two factors. The first one is the choice of interaction potential along the interface between the film and the substrate. In the generalized model, we assume that the potential energy is zero when $y \rightarrow \infty$; then the interface potential energy achieves the minimum at the equilibrium position $y=y_{0}$, while the interacting traction on the interface vanishes at the equilibrium state. At the initial stage, as an external force added on the film at $x=L$ increases from zero, the separation between the film and the substrate at $x=L$ will increase gradually. Meanwhile, the interacting traction on the interface will increase first and then decrease according to the LJ potential. In the approximate model, the adhesion energy of the interface is a constant $\Delta \gamma$ at the equilibrium state, i.e., $y=y_{0}$. At the initial stage, as an external force is exerted on the film at $x=L$, the interface will open suddenly only when the external force overcomes the interface strength. The external force at this moment is addressed as the peel-off force, with an interface separation larger than $y_{0}$. Along with the detachment of the interface, the angle $\theta_{L}$ increases gradually from zero to $\theta_{F}$, also leading to the decrease of the following peel-off force.

The second factor is due to different boundary conditions. In the approximate model, the film is divided into two segments: the left region $[0, l]$ of the film adhering to the rigid substrate perfectly with a constant interface adhesion energy $\Delta \gamma$ and the peeled-off region $[l, L]$ without considering the interface interaction. Because the bonding length $l$ is a variable that depends on the peeling force $F$, a boundary condition $\theta_{l}=0$ is used to obtain the length $l$ in the approximate model. Such a boundary condition looks like a fixed one. However, in the generalized model, no boundary condition is used and only an interacting potential works at $x=l$, which looks like a flexible boundary condition.

As a result, the approximate model can be reduced to the classical Kendall one (18), but it can only describe the steadystate peeling process and is invalid for the initial and transient regimes. Analogous to the approximate model, the classical Kendall one cannot predict the initial and transient peeling stages too.

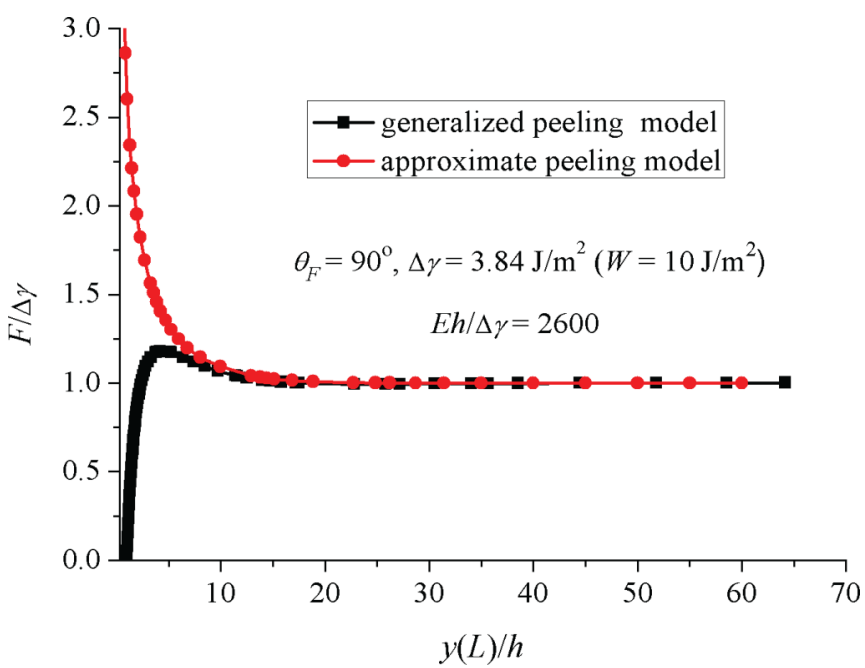

FIG. 2. (Color online) Comparison between the generalized peeling model and the simplified one with the same peeling angle $\theta_{F}=90^{\circ}$ and adhesion energy $\Delta \gamma=3.84 \mathrm{~J} / \mathrm{m}^{2}$. 

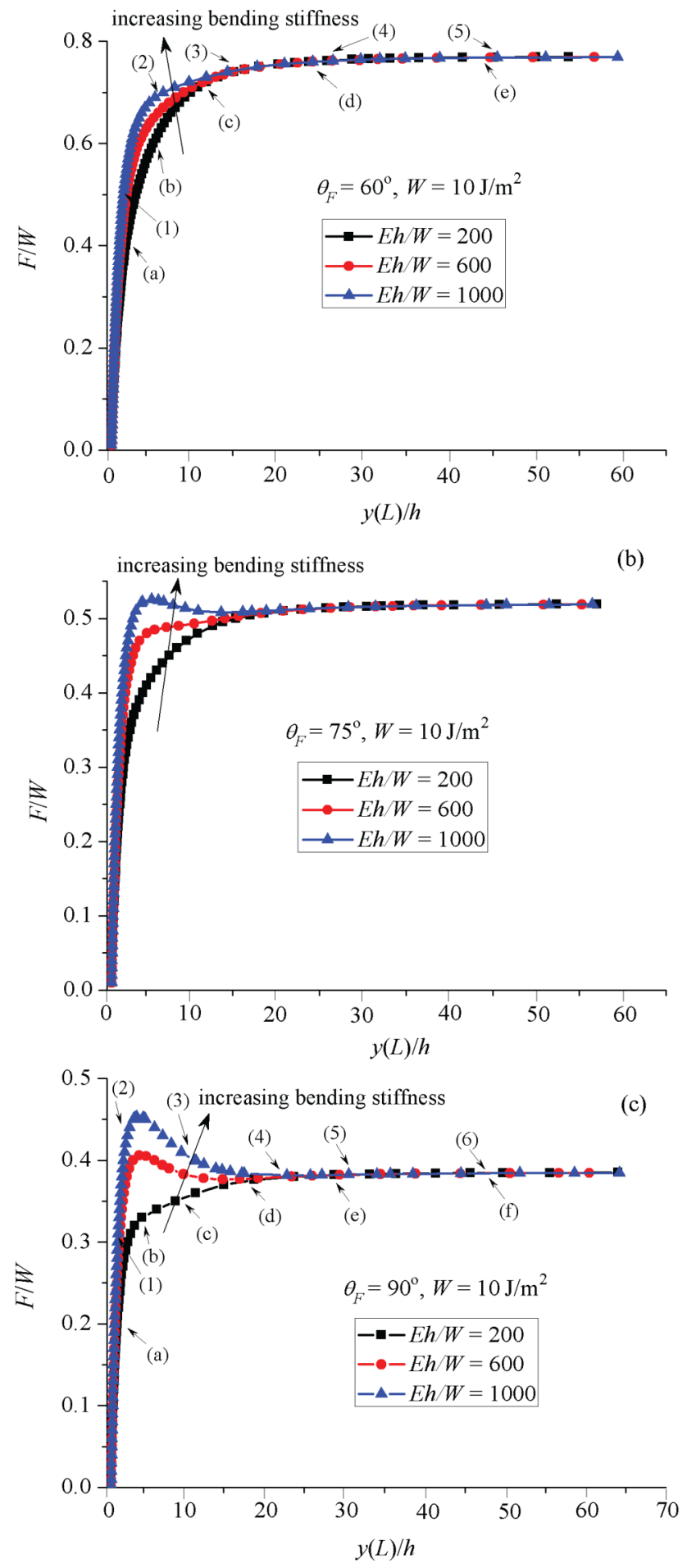

FIG. 3. (Color online) Peeling force varying with the peeling distance of the film with different bending stiffness for (a) $\theta_{F}=60^{\circ}$, (b) $\theta_{F}=75^{\circ}$, and (c) $\theta_{F}=90^{\circ}$. Labels of (i) in (a) and (c) denote different peeling moments, which will be used to compare with the corresponding snapshots in Figs. 5 and 6, respectively.

In view of the rationality analysis of the approximate model, all the following numerical calculations are performed according to the generalized one.

Figures 3(a)-3(c) show the typical peeling forcedisplacement relations with different peeling angles and film bending stiffness for a determined adhesion energy $W=10 \mathrm{~J} / \mathrm{m}^{2}$. One can see that the peeling angle and film bending stiffness influence significantly the peeling forcedisplacement relation (in the present paper, the film's thickness is kept a constant, thus the bending stiffness is only described by Young's modulus). Three representative values of peeling angles are discussed in Figs. 3(a)-3(c) with different bending stiffness of the film. In the case with a relatively small peeling angle, for example, $\theta_{F}=60^{\circ}$ as shown in Fig. 3(a), for each value of film bending stiffness, the peeling force increases monotonically first with the increase of the peeling distance and then remains constant when it reaches a steady-state peeling process. In contrast, in the case with a relatively large peeling angle, for example $\theta_{F}=75^{\circ}$ and $90^{\circ}$ as shown in Figs. 3(b) and 3(c), for the relatively small value of film bending stiffness, the peeling force increases monotonically first with the increase of the peeling distance and then remains constant when it reaches a steady-state peeling process; however, for the relatively large value of film bending stiffness, the peeling force increases first to a maximum and then decreases with the increase of peeling distance before a constant is achieved in a steady-state stage. It should be noted that the maximum peeling force is sometimes larger than the constant value achieved in a steady-state stage, which means that the interfacial peeling strength defined by the maximum peeling force does not always emerge at the steady-state stage, but significantly depends on the film bending stiffness and peeling angle. All the phenomena predicted theoretically in the present paper are consistent with the experimental and numerical results $[1,4,27]$.

From Fig. 3 one may also note that, for a fixed peeling angle and adhesion energy, the peeling force in the steady-state stage is insensitive to the bending stiffness. That is because the bending stiffness does not influence the steady-state peeling force since the bending energy does not change at the steadystate stage. However, Young's modulus plays a role in the steady-state peeling force due to the tension elastic energy, as predicted by Kendall's model. However, the effect of Young's

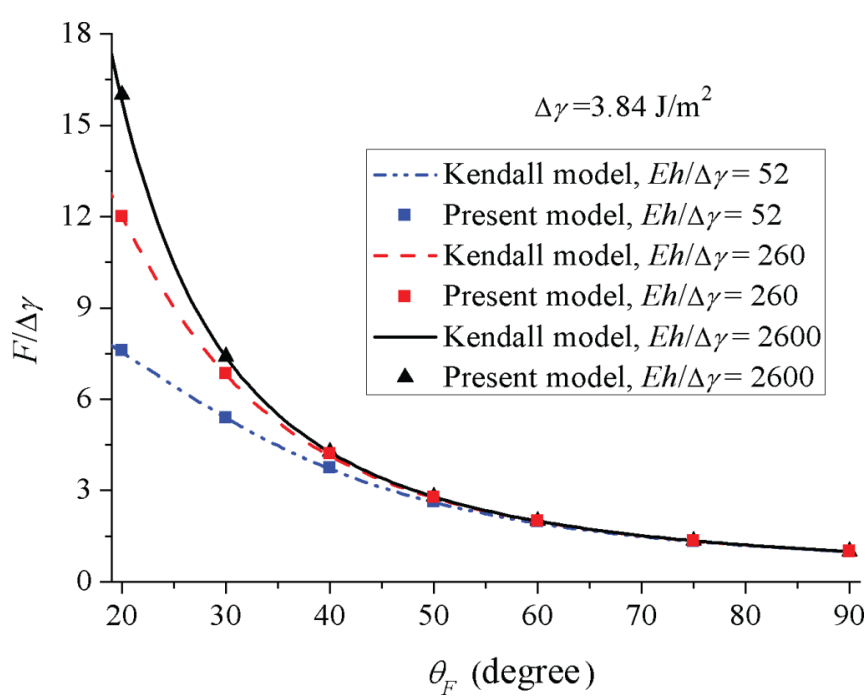

FIG. 4. (Color online) Comparison between the peeling force of the generalized model in the steady-state stage and that of Kendall's model with the same adhesion energy and Young's modulus. 


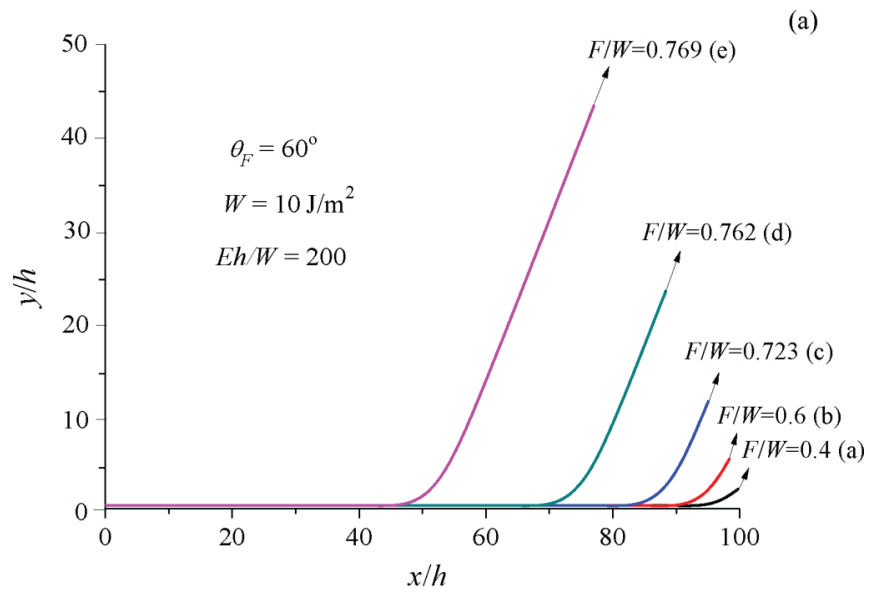

(b)

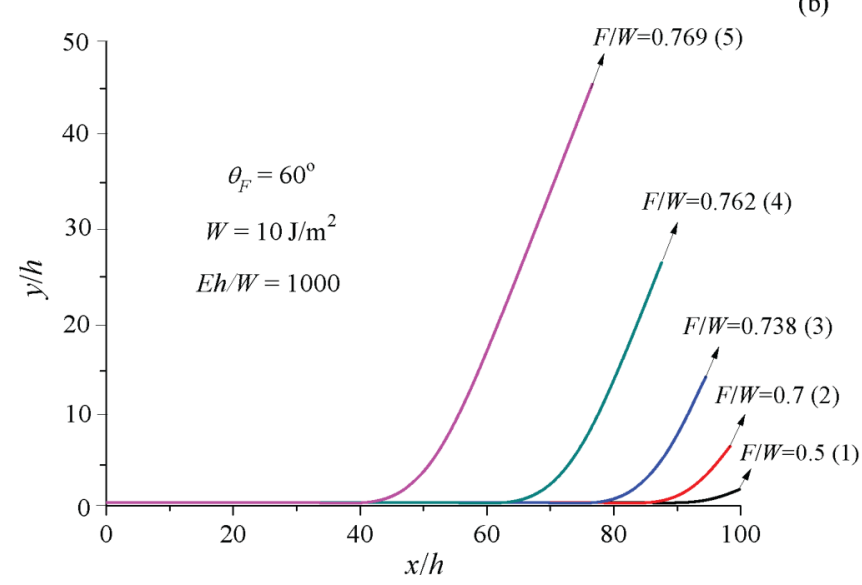

FIG. 5. (Color online) Profiles of the deformed film during different peeling moments with determined interface adhesion energy $W=10 \mathrm{~J} / \mathrm{m}^{2}$ and peeling angle $\theta_{F}=60^{\circ}$ for (a) $E h / W=200$ and (b) $E h / W=1000$. Labels on each profile correspond to those in Fig. 3(a).

modulus on the steady-state peeling force is very weak in cases with relatively large peeling angles, which has been pointed out by Kendall [9] and reappears in our results as shown in Fig. 4. It is found that the difference of the steady-state peeling force almost vanishes in cases with peeling angles varying from $60^{\circ}$ to $90^{\circ}$, though the magnitude of $E h / \Delta \gamma$ is significantly different. However, Young's modulus will show obvious influences on the steady-state peeling force if the peeling is less than $60^{\circ}$. In addition, one can see from Fig. 4 that the present theoretical results in the steady-state stage are consistent with those of Kendall's model.

At the initial peeling stage, why does the film's bending stiffness show significant effects on the peeling behaviors? Figures 5 and 6 give configurations of the deformed films with different bending stiffness in the whole peeling process under two peeling angles $\theta_{F}=60^{\circ}$ and $90^{\circ}$, which can help to understand the effect of the film's bending stiffness on the peeling behaviors. The varying peeling force for each snapshot is also correspondingly indicated in Fig. 3. It is shown that the deflection as well as the curvature of the film increases initially with the increase of the separation between the film and substrate, which results in the bending elastic energy stored in the film and the peeling force increasing. The higher the
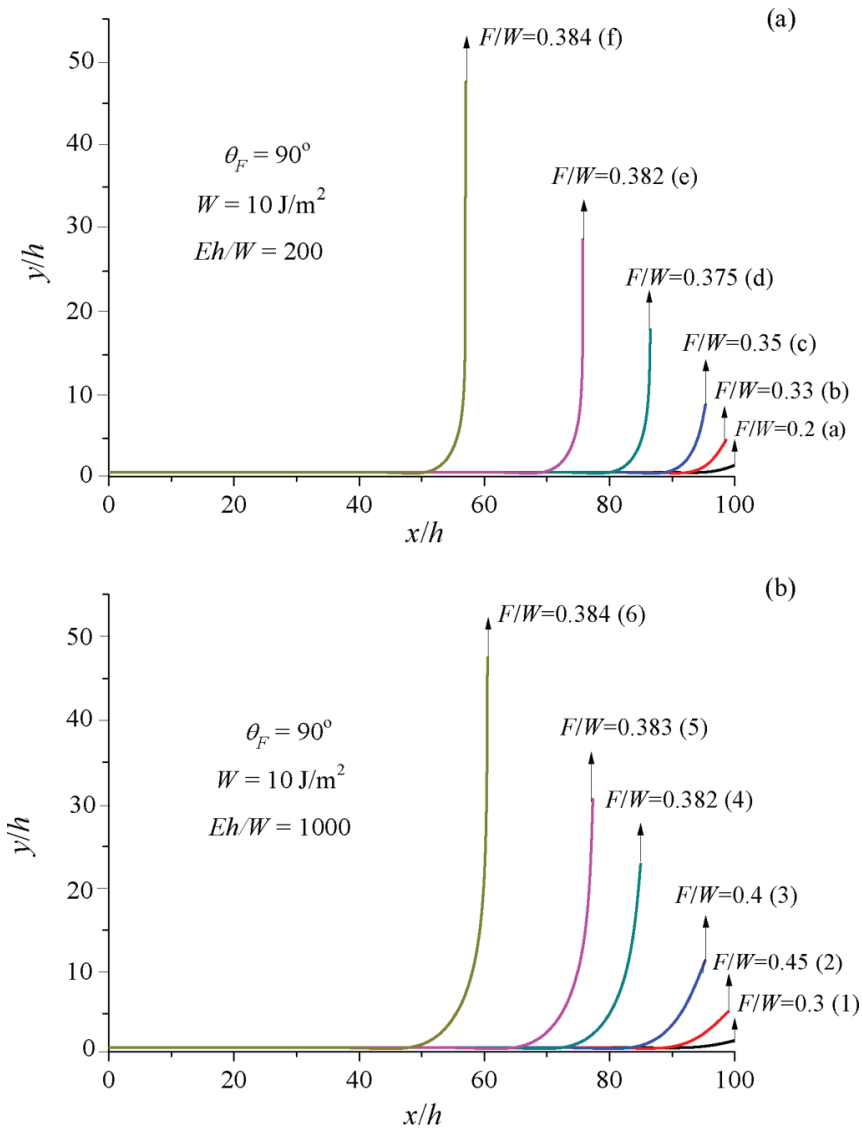

FIG. 6. (Color online) Profiles of the deformed film during different peeling moments with determined interface adhesion energy $W=10 \mathrm{~J} / \mathrm{m}^{2}$ and peeling angle $\theta_{F}=90^{\circ}$ for (a) $E h / W=200$ and (b) $E h / W=1000$. Labels on each profile correspond to those in Fig. 3(c).

bending stiffness is, the larger the peeling force will reach at the same separation due to larger bending energy stored in the film. When the separation attains a critical distance for a given peeling angle and film's bending stiffness, the film's end axis $\theta_{L}$ is consistent with the peeling direction $\theta_{F}$ and the profile of the film keeps a constant shape as shown in Figs. 5 and 6, after which the film reaches the steady-state peeling stage. In this case, the shape of the film does not change, so the bending elastic energy does not contribute to the total energy of the system and the peeling force remains constant during the steady-state peeling process. Comparing snapshots (2) and (3) in Fig. 6(b) to points labeled (2) and (3) on the curve in Fig. 3(c), we can find a turning point with a maximum value of the peeling force between them.

Figure 7 shows the peeling force varying with the separation of the loading point with a determined film's bending stiffness but different interface adhesion energy for a vertical peeling angle case, i.e., $\theta_{F}=90^{\circ}$. It is found that the peeling force increases monotonically with the increase of the separation of the loading point for relatively large interface adhesion energy; it increases to a maximum and then decreases to a constant at the steady-state stage for relatively small interface adhesion energy. During the steady-stage peeling process, the peeling force increases with increasing interfacial adhesion energy at a given peeling angle. 


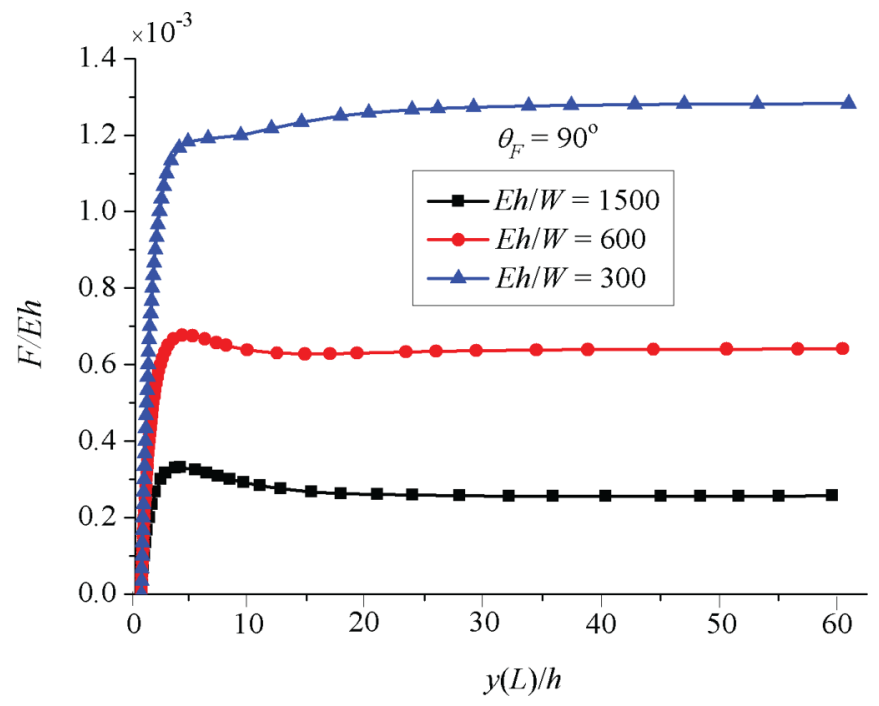

FIG. 7. (Color online) Peeling force varying with the peeling distance considering the effect of the adhesion energy with determined bending stiffness at $\theta_{F}=90^{\circ}$.

\section{CONCLUSION}

The principle of the minimum potential energy is used in this paper in order to achieve the whole peeling process of an elastic film on a rigid substrate. It is found that the bending stiffness of the film shows significant effects on the peeling force at the initial peeling stage. When the film reaches the steady-state peeling process, then bending elastic energy has no effect on the peeling force, but the tension elastic energy contributes to the peeling force to some extent, especially at relatively small peeling angles, which agrees well with Kendall's model. The maximum peeling force is sometimes larger than the one at the steady-state stage, especially for the film-substrate system with a relatively large film bending stiffness. Profiles of the deformed film during the whole peeling process are predicted, which can help explain the effect of the bending stiffness on the peeling force. It is found that the film's bending stiffness will influence the peeling force due to the increasing bending energy stored in the film until the tangential angle of the film at the loading point is consistent with the peeling angle. During the steady-state peeling process, the curvature of film remains the same, which leads to an identical bending energy as well as peeling force. Without considering the interface interaction in the detached region, the present model can be reduced to the classical Kendall model at the steady-state peeling process. The predictions of the present model disclose the whole peeling process of an elastic film with finite bending stiffness adhering to a rigid substrate, which agrees well with the existing experimental and numerical observations. The maximum peeling force can be predicted precisely no matter what the stiffness of film is, which should be helpful for the design and assessment of film-substrate interfaces in practical applications.

\section{ACKNOWLEDGMENTS}

The work reported here was supported by NSFC through Grants No. 11302228, No. 11125211, and No. 11372317 and the 973 Nano Project No. 2012CB937500.
[1] R. A. Sauer, J. Adhes. 87, 624 (2011).

[2] Y. G. Wei, Int. J. Solids Struct. 41, 5087 (2004).

[3] G. J. Spies, Aircr. Eng. Aerosp. Technol. 25, 64 (1953).

[4] J. Kim, K. S. Kim, and Y. H. Kim, J. Adhes. Sci. Technol. 3, 175 (1989).

[5] A. J. Kinloch, C. C. Lau, and J. G. Williams, Int. J. Fract. 66, 45 (1994).

[6] M. D. Thouless and H. M. Jensen, J. Adhes. 38, 185 (1992).

[7] H. Chen, X. Feng, Y. Huang, Y. G. Huang, and J. A. Rogers, J. Mech. Phys. Solids 61, 1737 (2013).

[8] P. Rahulkumar, A. Jagota, S. J. Bennison, and S. Saigal, Int. J. Solids Struct. 37, 1873 (2000).

[9] K. Kendall, J. Phys. D 8, 1449 (1975).

[10] M. J. Loukis and N. Aravas, J. Adhes. 35, 7 (1991).

[11] X. Feng, M. A. Meitl, A. M. Bowen, Y. Huang, R. G. Nuzzo, and J. A. Rogers, Langmuir 23, 12555 (2007).

[12] S. M. Xia, L. Ponson, G. Ravichandran, and K. Bhattacharya, J. Mech. Phys. Solids 61, 838 (2013).

[13] S. Xia, L. Ponson, G. Ravichandran, and K. Bhattacharya, Phys. Rev. Lett. 108, 196101 (2012).

[14] B. Chen, P. D. Wu, and H. J. Gao, J. R. Soc. Interface 6, 529 (2009).

[15] Z. L. Peng and S. H. Chen, Appl. Phys. Lett. 101, 163702 (2012).

[16] A. Molinari and G. Ravichandran, J. Adhes. 84, 961 (2008).

[17] N. S. Pesika, Y. Tian, B. X. Zhao, K. Rosenberg, H. B. Zeng, P. McGuiggan, K. Autumn, and J. N. Israelachvili, J. Adhes. 83, 383 (2007).
[18] Z. L. Peng, S. H. Chen, and A. K. Soh, Int. J. Solids Struct. 47, 1952 (2010).

[19] Y. Tian, N. Pesika, H. B. Zeng, K. Rosenberg, B. X. Zhao, P. McGuiggan, K. Autumn, and J. Israelachvili, Proc. Natl. Acad. Sci. USA 103, 19320 (2006).

[20] K. S. Kim and J. Kim, J. Eng. Mater. Technol. 110, 266 (1988).

[21] K. S. Kim and N. Aravas, Int. J. Solids Struct. 24, 417 (1988).

[22] Y. Wei and J. W. Hutchinson, Int. J. Fract. 93, 315 (1998).

[23] M. D. Thouless, M. S. Kafkalidis, S. M. Ward, and Y. Bankowski, Scr. Mater. 37, 1081 (1997).

[24] Z. X. Lu, S. W. Yu, X. Y. Wang, and X. Q. Feng, Eur. Phys. J. E 23, 67 (2007).

[25] M. D. Thouless and Q. D. Yang, Int. J. Adhes. Adhes. 28, 176 (2008).

[26] H. Yuan, J. F. Chen, J. G. Teng, and X. Z. Lu, Int. J. Solids Struct. 44, 5247 (2007).

[27] Y. G. Wei and H. F. Zhao, Int. J. Solids Struct. 45, 3779 (2008).

[28] X. Oyharcabal and T. Frisch, Phys. Rev. E 71, 036611 (2005).

[29] J. Israelachvili, Intermolecular and Surface Forces (Academic, London, 1991).

[30] M. P. de Boer and T. A. Michalske, J. Appl. Phys. 86, 817 (1999).

[31] J. L. Liu, X. Q. Feng, R. Xia, and H. P. Zhao, J. Phys. D 40, 5564 (2007). 